\title{
Shape Memory Effect of Benchmark Compliant Mechanisms Designed With Topology Optimization
}

\author{
Adrien Thabuis*, Sean Thomas*, Thomas Martinez and Yves Perriard ${ }^{1}$, Senior Member, IEEE
}

\begin{abstract}
Shape Memory Alloys (SMA) are a class of Smart Materials that are ideal for creating compact actuators. The complex nature of their behavior results in the use of these materials in simple geometries (wires, sheets) limiting their performances. A design method to develop complex compliant mechanisms made of SMA is proposed in this paper. Using topology optimization for conventional materials, a set of benchmark problems are designed. Finite element modelling is then able to validate the shape memory effect of the designs (with strain retention up to $80 \%$ ), confirming the notion that this methodology can, in fact, be used to design the next generation of SMA actuators.
\end{abstract}

Index Terms-smart material, topology optimization, computer-aided design, finite element analysis

\section{INTRODUCTION}

The modern age has brought about an increased demand for miniaturisation. Smaller, more high performing devices are required to meet this new trend.

Smart materials are a new class of materials that react mechanically to a certain kind of stimuli such as heat, electric current or a magnetic field. Among them, Shape Memory Alloys (SMA) are one kind that reacts to heat. These alloys have become increasingly popular when designing compact actuators that can still provide large amounts of work. The work by Brinson [1] demonstrates a constitutive model for the Shape Memory Effect (SME) which shows the dependencies of the various internal variables and material constants. The complexity of the behavior makes it difficult to create actuators that can harness its exotic properties. Generally, the most common method to implement SMA actuators would be to work with the material in simple shapes such as wires [2], [3], springs [4] or sheets [5]. The use of simple shapes stems from the fact that the complex behavior of the SMA is hard to model and design.

Another promising avenue for creating compact and efficient actuators is the rise of compliant mechanisms. They transform an input displacement or force into an output one through elastic deformation. Admittedly, the permitted stroke is limited but friction with other parts is removed, improving the overall lifetime of the mechanism and providing better precision at the output. Composed of a single part, a compliant mechanism fulfils a function that is alternatively satisfied with multiple pieces, reducing the overall complexity of assembly all the while remaining compact. The design of such elastic mechanisms is a tedious task that can be

\footnotetext{
*These authors contributed equally to this work

${ }^{1}$ These authors are with the Integrated Actuators Laboratory, Ecole Polytechnique Fédérale de Lausanne (EPFL), Switzerland yves.perriardeepfl.ch
}

accomplished in two different ways. The first one is the conventional design approach that relies on the designer's experience and mathematical models. It essentially divides the design problem into the sizing of systems composed of rigid links and flexural joints [6]. The second one includes various computational-aided methods that formulate the design problem as an optimization one. Among them, the so-called Topology Optimization Method (TOM) has been subject to a large research interest over the past years [7], [8]. It is a powerful and versatile design approach that directly investigates the distribution of material inside a discretized design space all the while improving an objective.

The behavior of SMAs created in intricate shapes such as compliant mechanisms are difficult to predict and are not certain to perform in an intended way and thus most work in the field has avoided combining compliant mechanisms and SMAs. This work will attempt to design novel SMA Compliant Mechanisms and show that they do in fact show the shape memory effect in the same order of magnitude as ones created in simple structures.

Instead of explicitly integrating the SME into the design process, topology optimization of compliant mechanisms made of standard elastic materials is performed. This allows to strongly reduce the overall computational time. The density-based approach TOM is selected in this project as it is the most robust one and has already been massively investigated for the design of compliant mechanisms [9][11].

As a result of the natural complementarity of compliant mechanisms and SMAs, the abstraction of their thermal properties during the design process does not affect the actual shape memory effect of the mechanism. The behavior of the designed actuators is validated using commercially available Finite Element Modelling (FEM) software such as ANSYS.

\section{SHAPE MEMORY EFFECT}

SMAs are an interesting type of Smart Material that reacts to thermal loads. These materials, most commonly used being NiTiNOL (an alloy of Nickel and Titanium), shows two very unique behaviours: the Shape Memory Effect (SME) and Superelasticity (SE). These behaviours are a manifestation of the same property which consists of the material reverting back to its original shape when its temperature exceeds a certain threshold. The unique behaviour exists due to the change in phases of the material at different temperatures. In Fig. 1, the different phases and phase transformations are demonstrated. The material exists in 3 phases: the twinned and detwinned Martensite (M) phase at low temperatures and 


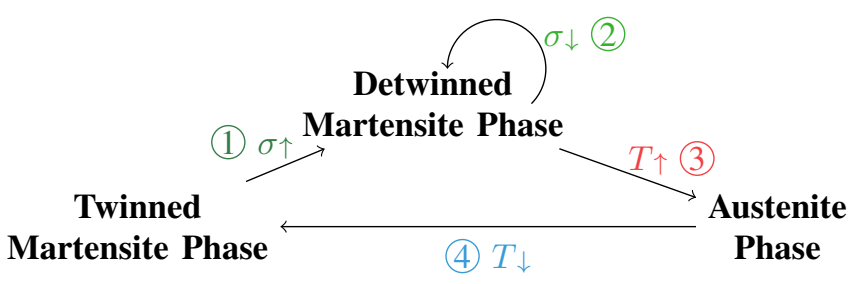

Fig. 1: Phase transformations of Shape Memory Alloys depending on the applied stress $\sigma$ and temperature $T$.

the Austenite (A) phase at high temperatures. As seen in Fig. 1, the material, at low temperatures (1), will exist in the twinned $\mathrm{M}$ phase. When it is then exposed to a stress, higher than a certain critical stress $\left(\sigma_{s}^{c r}\right)$, it will transform into the detwinned $M$ phase. When the stress is then removed (2), the material will stay in this detwinned $M$ phase and the strain observed by the material will remain. This phase transformation is important, as when the material is then heated above its transformation temperature and transforms into its A phase (3), only the sections of the material that has been transformed to the detwinned $\mathrm{M}$ phase will undergo the strain recovery and revert back to their original shape. In essence, the material will have to undergo a plastic deformation at low temperatures to be considered activated. Only then, will the material release back the energy in the form of strain recovery, when it is heated.

Due to the complex behaviour of the SMA, most actuators that harness this material make use of it in simple geometries such as wires, springs and sheets. The general idea when it comes to implementing an SMA actuator is to attach the SMA wire or spring to a biasing element such as a spring, dead weight or another SMA element so as to deform the SMA at low temperatures to activate the material. In the case of a bias-spring, the spring will deform the SMA at low temperatures and alternatively, at high temperatures, the SMA will deform the bias-spring resulting in a reversible actuation.

\section{DESIGN METHOD}

As previously introduced, shape memory alloys have complex multi-physics non-linear material properties. Simulating their behavior with finite element software is computationally heavy even with poor mesh resolution. To design compliant mechanisms made of SMAs with computer-aided methods like TOM, the authors choose to do abstraction of the temperature-dependent effects but appropriately formulate the design problem so as to indirectly take into account their multi-physics properties. By optimizing purely for strain and assuming standard elastic material, various compliant mechanisms can be designed while still indirectly taking into account the SME of the desired mechanism. Indeed, as long as the material is undergoing enough deformation, enough strain, its shape memory properties will be activated. The analysis is performed assuming small and purely elastic deformation to rely on linear finite element analysis and reduce significantly the computational time.

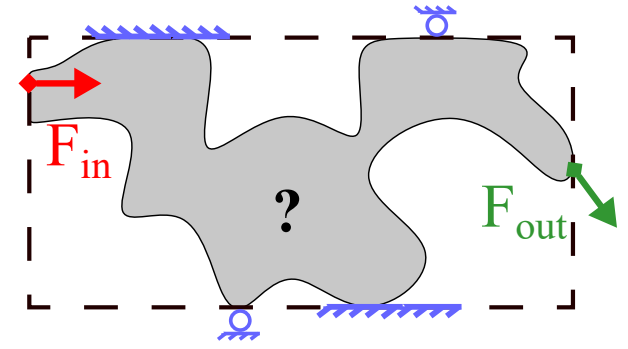

Fig. 2: Design Problem Compliant Mechanism

\section{A. Density-Based Topology Optimization}

Topology optimization investigates the distribution of material inside a discretized design domain that improves a specified objective function. The design variables used in the density-based approach are artificial densities $\rho$ that describe the proportion of material $0<\rho_{\min } \leq \rho \leq 1$ an element of the discretized design domain is filled in with. The lower $\rho_{\text {min }}$ and upper 1 bounds are qualified as full materials representing fully empty or fully filled elements respectively, as opposed to the other values defined as intermediate materials. The aforementioned design variables are made continuous to use gradient-based solvers which will speed significantly the overall computational time compared to non-gradient-based approaches [12]. In order to avoid mesh-dependent solutions and checkerboard patterns, regulation of the optimization is required. It is achieved with a density filter that imposes a minimal length scale $r_{\min }$ inside the structure [7].

The following steps are performed at each iteration of the optimization. The filtered design variables are mapped to material properties through the Material Interpolation Scheme (MIS) which converts the artificial densities to young moduli. The famous SIMP (Solid Isotropic Material Penalization) is implemented in this research and showed in (1) [7]. This scheme also penalizes intermediate materials so as to have a manufacturable final topology composed of only full materials. It is done through the penalty factor $n$ that is continuously increased throughout the optimization.

$$
\mathrm{E}_{e}=\operatorname{MIS}\left(\rho_{e}\right)=(\overline{\mathrm{E}}-\underline{\mathrm{E}}) \rho_{e}^{n}+\underline{\mathrm{E}}
$$

with $\underline{E}, \overline{\mathrm{E}}$ the lower and upper bound of $\mathrm{E}=\left[\mathrm{E}, \cdots, \mathrm{E}_{\mathrm{NE}}\right]^{\mathrm{T}}$ respectively corresponding to the Young modulus of an "empty" element and a completely filled one. NE is the number of elements inside the domain. Once the material properties of each element are known, the Stiffness matrix $\mathrm{K}$ of the corresponding structure is built. Finite Element Analysis (FEA) is performed to evaluate the displacement $\mathrm{U}$ of each node under imposed external loads F. It solves the linear elasticity equation: $\mathrm{KU}=\mathrm{F}$. These displacements are used to evaluate the chosen objective function as well as its associated sensitivity (gradient of the objective function with respect to the design variables). This sensitivity is used by the solver to update the design variables for the next iteration. The implemented solver corresponds to the Optimality Criteria (OC) scheme [7]. 

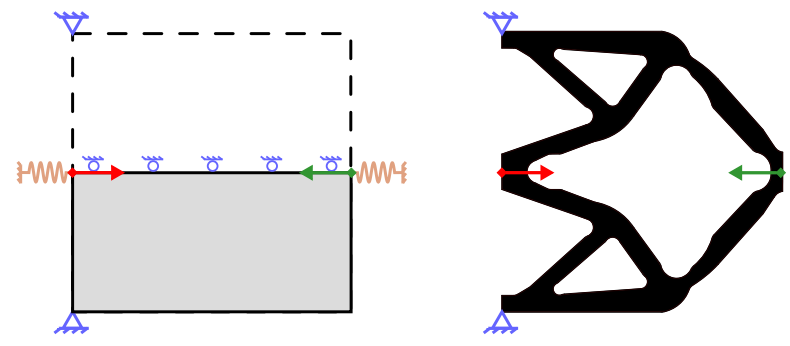

Fig. 3: Inverter: Design problem (left), Interpolated final topology (right). Input force is shown in red while the output force is shown green.
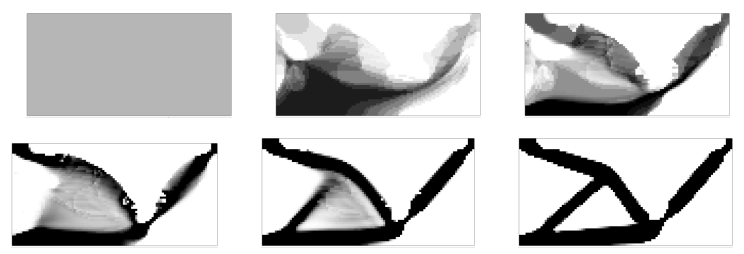

Fig. 4: Inverter: Evolution throughout the optimization. The grayscale represent the value of the filtered artificial density.

Various academic codes performing structural topology optimization in Matlab are available [8], [13]. The encoded physics/FEA being the same as compliant mechanisms, one can easily extend them by only changing the objective function and the associated sensitivity, if not already available.

\section{B. Topology Optimization of Compliant Mechanisms}

The general design problem of compliant mechanisms is illustrated in Fig. 2 and can be formulated as the following question: "Under an external input force and certain boundary conditions, what is the distribution of material defining the compliant mechanism that transforms the input load to a specific output one?"

Artificial springs are added at the input and output ports to simulate the input work and the resistance to the output displacement of the workpiece, respectively [9]. They are just used during the design and do not imply the necessity of springs for the practical integration of the compliant mechanism.

As for the choice of the objective function that governs the design process of compliant mechanisms, various formulations have already been proposed [8], without significant differences for simple problems composed of single input and output. As this research will later be extended to the design of more complex problems composed of Multi-Inputs MultiOutputs (MIMO), a formulation based upon various strain energies that is easily extended to such problems is chosen [9]. Three different types of strain energies are used to build the objective function. There is the input strain energy $S_{\text {in }}$, that is present when only an input load $F_{i n}$ is applied and result in some nodal displacements $U_{\text {in }}$. Similarly, the output strain energy $S_{\text {out }}$, is present when only a dummy unit load $\mathrm{F}_{\text {out }}$ is applied at the output port resulting in nodal displacements $U_{\text {out }}$. The Mutual Potential Energy (MPE) or mutual strain energy $S_{\text {mut }}$ can be built with the two previous nodal

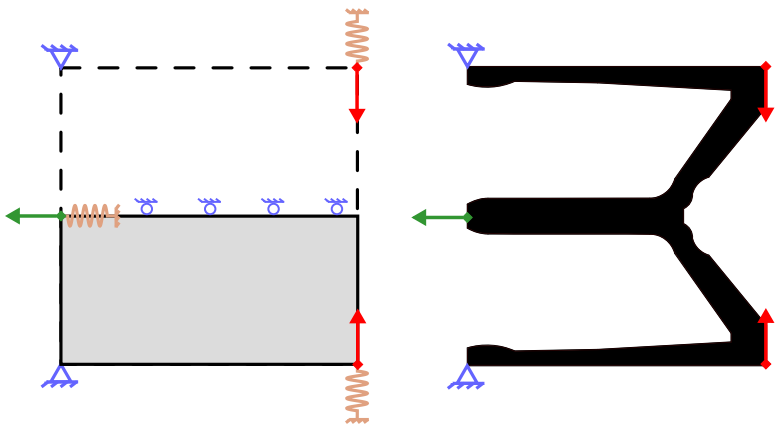

Fig. 5: Crimper: Design problem (left), Interpolated final topology (right). Input force is shown in red while the output force is shown green.
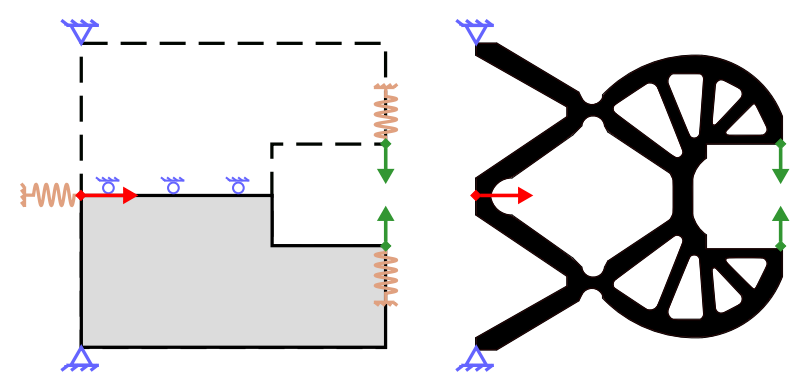

Fig. 6: Gripper: Design problem (left), Interpolated final topology (right). Input force is shown in red while the output force is shown green.

displacements. A ratio combining these energies is chosen as the objective function due to its non-dimensional aspect. The desired input and output displacements are indirectly accounted for with this objective [10]. The problem being formulated as a minimization one, a minus sign is placed in front of the chosen ratio to maximize the mutual potential energy:

$$
f=-\frac{\mathrm{S}_{\mathrm{mut}}}{\mathrm{S}_{\mathrm{in}}+\mathrm{S}_{\text {out }}}=-\frac{\mathrm{U}_{\text {out }}{ }^{\mathrm{T}} \mathrm{KU}_{\text {int }}}{\frac{1}{2} \mathrm{U}_{\text {out }}{ }^{\mathrm{T}} \mathrm{KU}_{\text {out }}+\frac{1}{2} \mathrm{U}_{\mathrm{in}}{ }^{\mathrm{T}} \mathrm{KU}_{\mathrm{in}}} .
$$

Two separate FEA have to be performed to evaluate the chosen objective: $\mathrm{KU}_{\text {in }}=\mathrm{F}_{\text {in }}$ and $\mathrm{KU}_{\text {out }}=\mathrm{F}_{\text {out }}$.

This objective governing the design of compliant mechanism made of conventional material, happens to already be a good one for mechanisms made of SMA. Indeed, it will distribute the strain throughout the structure which will indirectly activate the material if made in SMA. Ideally, the maximum amount of the structure should be deformed to harvest as much work, as much shape memory effect as possible.

The optimization problem is formulated in (3) as the minimization of the objective function $f$ subject to the limitation of the infill $\mathrm{Vol}^{*}$ of the design domain's volume, with $V_{e}$ the volume of element $e$. 


$$
\begin{aligned}
\underset{\rho}{\min }: & f\left(\mathrm{U}_{\text {in }}, \mathrm{U}_{\text {out }}, \mathrm{E}\right) \\
\text { s.t. : } & \mathrm{KU}_{\text {in }}=\mathrm{F}_{\text {in }} \\
& \mathrm{KU}_{\text {out }}=\mathrm{F}_{\text {out }} \\
& \mathrm{Vol}<\mathrm{Vol}^{*}, \text { with } \mathrm{Vol}=\left(\sum_{e=1}^{\mathrm{NE}} \rho_{e} \cdot V_{e}\right) /\left(\sum_{e=1}^{\mathrm{NE}} V_{e}\right) \\
& \mathrm{E}=\operatorname{MIS}(\boldsymbol{\rho}) \quad 0<\rho_{\min } \leqslant \rho \leqslant 1 .
\end{aligned}
$$

A specific case of benchmark compliant mechanisms is the force inverter. Fig. 3 shows the design problem with the final topology resulting from the topology optimization. As the problem is symmetric, the investigation of material's distribution can be restricted to the lower half if rolling supports are imposed as boundary conditions on the symmetry axis. The evolution of the half-topology throughout the optimization is shown in Fig. 4. The design domain is uniformly initialized with intermediate materials satisfying the volume limitation and will converge to a solution composed of only full materials for the final topology.

\section{Validation of the Shape Memory Effect}

The resulting designs obtained through topology optimization are quite complex and predicting the behaviour of the structure made from SMA is quite difficult. But, with the help of the commercially available FEM software, ANSYS [14], the behaviour of the designs can be simulated and compared.
The simulation consists of 4 time-steps which can be compared to Fig. 1 and the Figs. 7 to 9 whose times steps are labelled in the same manner. In the first time step, the appropriate boundary conditions are placed on the structure. The input displacement is applied during this first step and the output displacement is observed at the appropriate location (1). During the second time step, the input displacement is released and the retention of the displacement is observed (2). During this phase, if the structure reverts to its original shape (without heating), it implies that the SMA has not undergone the detwinning. This indicates that the material is not activated and thus no shape memory effect is observed. Only the areas of the structure that have undergone detwinning (whose strain has been retained) will revert back to its original shape when heated. The percentage of the material or the displacement that does not revert to the original shape can be used as a quantitative factor for comparing the ability of the design to be fabricated as an SMA actuator. At the next step, the structure is heated to revert the design back to its original shape and observe the strain recovery of the SME (3). Lastly, the structure is cooled down to return the structure to its initial phase (4).

\section{RESULTS}

In this paper, we validate the proposed approach by the study of benchmark problems used in the design of compliant mechanisms through topology optimization. The shape memory effect is then studied in a non-linear (FEA)
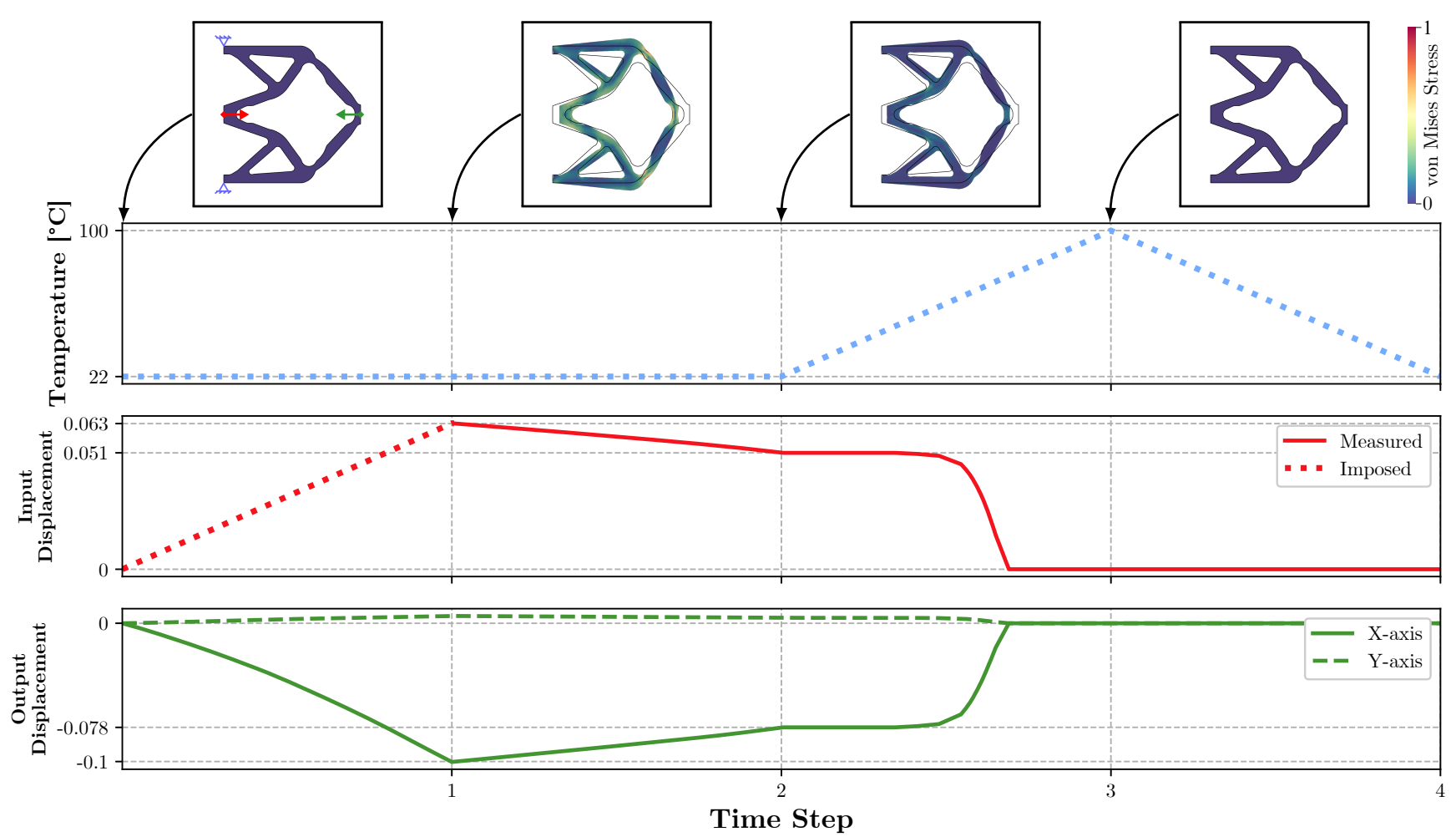

Fig. 7: Results of the shape memory effect simulation of the Inverter design. Here, the displacements are calculated relatively to the design domain size $L$. The displacements observed between time steps 1 and 2 show a strain retention of $\alpha_{\varepsilon}=79.9 \%$ confirming the presence of the shape memory effect. 

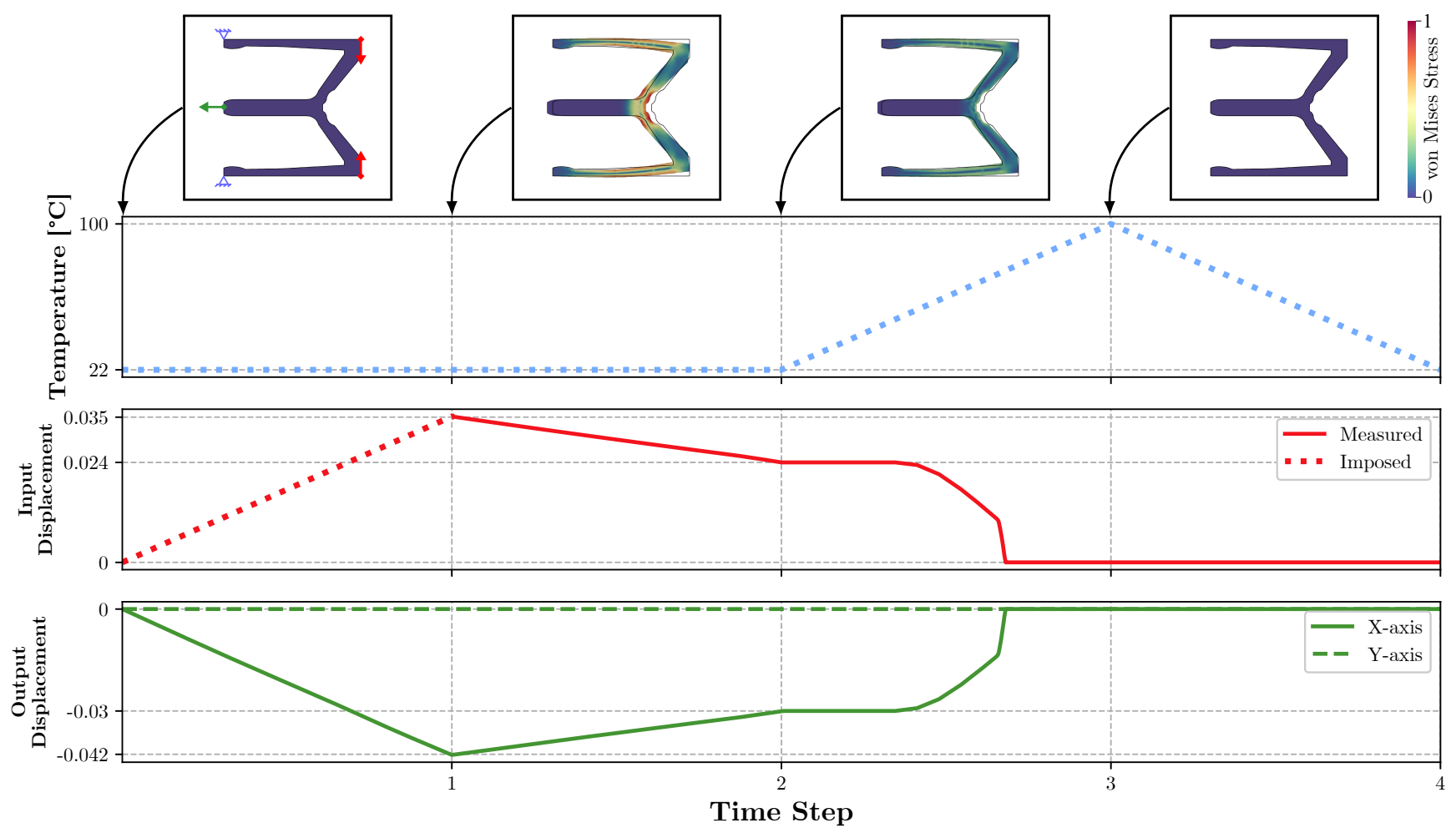

Fig. 8: Results of the SME simulation of the Crimper design. Here, the displacements are calculated relatively to the design domain size $L$. The displacements observed between time steps 1 and 2 show a strain retention of $\alpha_{\varepsilon}=68.8 \%$ confirming the presence of the shape memory effect.

performed with the ANSYS software. The SME observed for the benchmark problems are compared using the strain retention:

$$
\alpha_{\varepsilon}=1-\frac{\varepsilon_{\text {loaded }}-\varepsilon_{\text {free }}}{\varepsilon_{\text {loaded }}},
$$

with $\varepsilon_{\text {loaded }}$ and $\varepsilon_{\text {free }}$ representing the domain space normalised input displacement at time step 1 and 2 respectively. The factor $\alpha_{\varepsilon}$ varies between 0 and 1 and is used to compare the different benchmark problems. Here, if $\alpha_{\varepsilon}=0$, it implies that no SME has been observed and the structure will not react to heat. Contrarily, if $\alpha_{\varepsilon}=1$, it implies that all the parts of the structure contributing to the output displacement have been detwinned and will return to their original shape only when heated.

The topology optimization framework is implemented in Matlab. The studied problems have squared design domain of size $L \times L$. The following parameters have been fixed for all the presented results: $\rho_{\text {min }}=1 e^{-3}, \mathrm{Vol}^{*}=30 \%$, $\mathrm{NE}=10000, r_{\min }=L / 10$.

The topology resulting from the optimization does not have smooth boundaries due to the design process. Indeed the grid mesh degrades the resolution of the topology, resulting in a pixel-like structure. As much as the simulation software can import several formats, if the solid is defined by a large number of vertex without smooth boundaries, the routine that generates the mesh will struggle to proceed or result in poor mesh quality, degrading the convergence and the results of the simulation. To counter this, the boundaries are smoothed, interpolated using CAD software. As this step may change the topology, biasing the previously obtained results, the authors recommend to not fully automate this step and proceed with care on smoothing some parts of the boundaries step by step.

The results pertaining to the Force Inverter problem can be seen in Figs. 3, 4 and 7 showing a strain retention $\alpha_{\varepsilon}$ of $79.9 \%$. Similarly, Figs. 6 and 9 shows the results of the Gripper problem showing a strain retention of $74.5 \%$. Lastly, the results for the Crimper problem can be referenced in Fig. 5. The results of the simulation are shown in Fig. 8, its strain retention factor $\alpha_{\varepsilon}$ was found to be $68.8 \%$.

\section{DISCUSSION}

The results show that the benchmark problems when simulated, based on the strain retention factor, have a large amount of the shape memory effect. This implies that when heating the structure, the strain that was induced in the initial time steps can be recovered to create a viable SMA smart actuator.

The design is performed assuming small deformation and linear elastic material properties. Integrating large deformation FEA inside the topology optimization process could improve the obtained solutions, but will naturally degrade the computational time.

The compliant mechanisms obtained are still composed of some partial hinges, resulting in non-fully distributed vonMisses stresses inside the structure. The design of hinge-free 

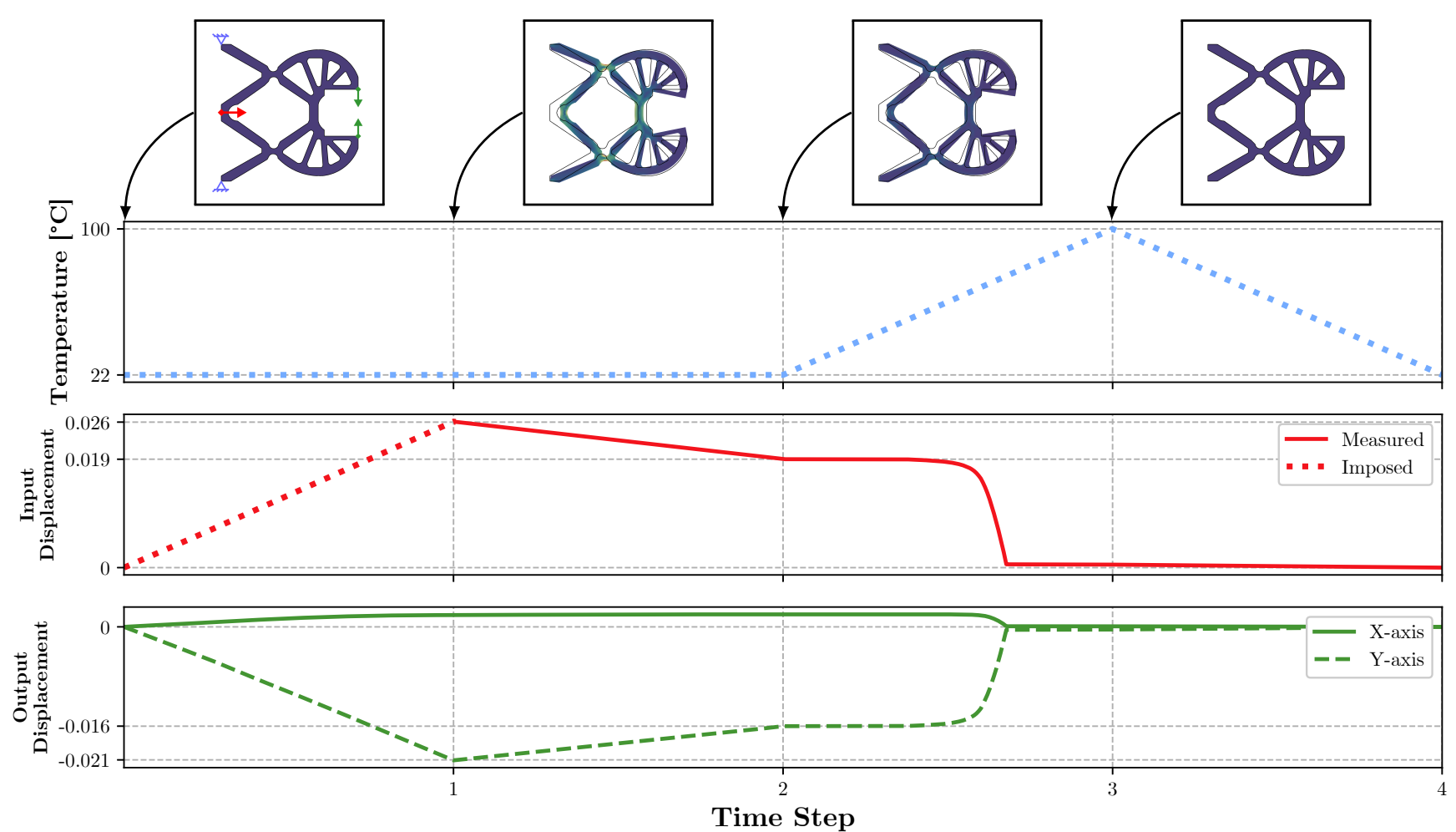

Fig. 9: Results of the SME simulation of the Gripper design. Here, the displacements are calculated relatively to the design domain size $L$. The displacements observed between time steps 1 and 2 show a strain retention of $\alpha_{\varepsilon}=74.5 \%$ confirming the presence of the shape memory effect. The outputs are symmetrical and thus, only the displacement of one is shown.

mechanisms is currently undergoing large research interest, but no robust solution seems to have been achieved yet [8]. Such mechanisms could further improve the SME of the proposed designs as more material would reach the critical detwinning stress.

\section{CONCLUSION}

In this work, the authors were able to introduce a type of design method that allows for the design of novel Shape Memory Alloy structures using Topology Optimization. Due to the complex nature of the shape memory effect, most work regarding SMAs focuses on simple geometries. Here, the designs generated using Topology Optimization, confirmed using FEM simulation, still show around $75 \%$ of the Shape Memory Effect. This work validates the idea that pairing Compliant Mechanisms with SMAs can result in compact and effective Smart Actuators.

\section{REFERENCES}

[1] L. Brinson, "One-Dimensional Constitutive Behavior of Shape Memory Alloys: Thermomechanical Derivation with Non-Constant Material Functions and Redefined Martensite Internal Variable," Journal of Intelligent Material Systems and Structures, vol. 4, no. 2, pp. 229242, Apr. 1993.

[2] J.-H. Lee, Y. S. Chung, and H. Rodrigue, "Long Shape Memory Alloy Tendon-based Soft Robotic Actuators and Implementation as a Soft Gripper," Scientific Reports, vol. 9, no. 1, pp. 1-12, Aug. 2019.

[3] R. Britz, P. Motzki, and S. Seelecke, "Scalable Bi-Directional SMABased Rotational Actuator," Actuators, vol. 8, no. 3, p. 60, Aug. 2019.
[4] Z. Zhakypov, F. Heremans, A. Billard, and J. Paik, "An OrigamiInspired Reconfigurable Suction Gripper for Picking Objects With Variable Shape and Size," IEEE Robotics and Automation Letters, vol. 3, no. 4, pp. 2894-2901, Oct. 2018

[5] R. Vitushinsky, S. Schmitz, and A. Ludwig, "Bistable Thin-Film Shape Memory Actuators for Applications in Tactile Displays," Journal of Microelectromechanical Systems, vol. 18, no. 1, pp. 186-194, Feb. 2009.

[6] L. L. Howell, Handbook of Compliant Mechanisms. John Wiley \& Sons, Ltd, 2013.

[7] M. P. Bendsøe and O. Sigmund, Topology optimization: theory, methods, and applications, ser. Engineering online library. Berlin Heidelberg: Springer, 2011, oCLC: 711862335.

[8] B. Zhu, X. Zhang, H. Zhang, J. Liang, H. Zang, H. Li, and R. Wang, "Design of compliant mechanisms using continuum topology optimization: A review," Mechanism and Machine Theory, vol. 143, p. 103622, Jan. 2020.

[9] C. Alonso, R. Ansola, and O. M. Querin, "Topology synthesis of MultiInput-Multi-Output compliant mechanisms," Advances in Engineering Software, vol. 76, pp. 125-132, Oct. 2014.

[10] A. Krishnakumar and K. Suresh, "Hinge-Free Compliant Mechanism Design Via the Topological Level-Set," J. Mech. Des, vol. 137, no. 3, Mar. 2015.

[11] B. Zhu, Q. Chen, H. Li, H. Zhang, and X. Zhang, "Design of Planar Large-Deflection Compliant Mechanisms With Decoupled MultiInput-Output Using Topology Optimization," J. Mechanisms Robotics, vol. 11, no. 3, p. 031015, Apr. 2019.

[12] O. Sigmund, "On the usefulness of non-gradient approaches in topology optimization," Struct Multidisc Optim, vol. 43, no. 5, pp. 589-596, May 2011.

[13] C. Talischi, G. H. Paulino, A. Pereira, and I. F. M. Menezes, "PolyTop: A Matlab implementation of a general topology optimization framework using unstructured polygonal finite element meshes," Struct Multidisc Optim, vol. 45, no. 3, pp. 329-357, Mar. 2012.

[14] P. Terriault, F. Viens, and V. Brailovski, "Non-isothermal finite element modeling of a shape memory alloy actuator using ANSYS," Сотриtational Materials Science, vol. 36, no. 4, pp. 397-410, Jul. 2006. 